\title{
Geographic Resource Allocation Based on Cost Effectiveness: An Application to Malaria Policy
}

\author{
Tom L. Drake ${ }^{1,2,3} \cdot$ Yoel Lubell $^{2,4} \cdot$ Shwe Sin Kyaw $^{1} \cdot$ Angela Devine $^{4}$. \\ Myat Phone Kyaw $^{5}$ - Nicholas P. J. Day ${ }^{2,4}$ - Frank M. Smithuis ${ }^{1,2} \cdot$ \\ Lisa J. White ${ }^{2,4}$
}

Published online: 10 February 2017

(c) The Author(s) 2017. This article is published with open access at Springerlink.com

\begin{abstract}
Healthcare services are often provided to a country as a whole, though in many cases the available resources can be more effectively targeted to specific geographically defined populations. In the case of malaria, risk is highly geographically heterogeneous, and many interventions, such as insecticide-treated bed nets and malaria community health workers, can be targeted to populations in a way that maximises impact for the resources available. This paper describes a framework for geographically targeted budget allocation based on the principles of cost-effectiveness analysis and applied to priority setting in malaria control and elimination. The approach can be used with any underlying model able to estimate intervention costs and effects given relevant local data. Efficient geographic targeting of core malaria interventions could significantly increase the impact of the resources available, accelerating progress towards elimination. These methods may also be applicable to priority setting in other disease areas.
\end{abstract}

Tom L. Drake

tom.d@tropmedres.ac

1 Myanmar Oxford Clinical Research Unit, Yangon, Myanmar

2 Nuffield Department of Medicine, University of Oxford, Oxford, UK

3 Faculty of Tropical Medicine, Mahidol-Oxford Tropical Medicine Research Unit, Mahidol University, 3/F, 60th Anniversary Chalermprakiat Building, 420/6 Rajvithi Road, Bangkok, Thailand

4 Mahidol-Oxford Tropical Medicine Research Unit, Mahidol University, Bangkok, Thailand

5 Department of Medical Research, Ministry of Health, Yangon, Myanmar

\section{Key Points for Decision Makers}

Geographic heterogeneity in disease risk and other factors is important to malaria control and elimination policy.

Yet geographic heterogeneity does not feature prominently in the malaria economic evaluation evidence base.

This paper describes an approach to geographic allocation of a malaria budget based on cost effectiveness.

\section{Introduction}

Effective spending is critical to malaria control and elimination. While global financing for malaria has risen considerably in recent years [1], it is not possible to provide all malaria interventions to all areas at risk. In addition to ensuring sustained financial and political commitment, policy makers must determine how best to marshal the available resources to maximise impact on malaria burden. Economic evaluation aims to address this question by providing information on the health impact of interventions in proportion to the resources required.

Several reviews have sought to summarise the evidence base on the cost effectiveness of malaria interventions [2-4]. This evidence has most clearly informed malaria policy when an intervention was shown to be highly cost effective in a wide range of settings, supporting generalisable policy recommendations. Examples include the use 
of artesunate for the treatment of severe malaria [5] and the introduction of point-of-care or rapid diagnostic tests [6-8]. However, translating the cost-effectiveness evidence base to inform detailed malaria control and elimination planning can be challenging, as described in an accompanying paper.

\subsection{The Importance of Geographic Variation in Malaria Policy}

The national malaria control programme manager is faced with, among other things, a toolbox of interventions, a limited budget and a map of malaria risk. With increasingly abundant geo-data on malaria incidence and the use of spatial-statistical models to fill in the gaps [9-11], malaria risk maps are increasingly useful and becoming more popular as there is growing recognition of the high degree of heterogeneity in risk within countries, within provinces or districts, and even within communities [12]. Related to this, most malaria interventions are highly divisible, down to the community or patient level, and can be targeted to specific geographically defined populations. These interventions include insecticide-treated bed nets (ITNs), chemoprevention, indoor residual spraying, larviciding, early detection and treatment through malaria community health workers (CHW), mass treatment and, perhaps soon, vaccination.

In addition to disease risk, further factors that vary with geography are relevant to malaria programming, such as access to treatment and the higher cost of delivering services in remote areas, though the cost of such services may well be considered worthwhile. In many countries, efforts are being made to identify the human and ecological dynamics behind geographic heterogeneity in malaria risk $[13,14]$; trials are underway to identify packages of interventions that could successfully target malaria hotspots [15] and spatial decision-support systems are being established [16, 17]. Locating malaria risk and deciding how to respond to this risk is therefore essential to effective control and elimination.

\subsection{Geographic Heterogeneity and Economic Evaluation}

While geographic heterogeneity is clearly important to malaria policy making, it does not feature prominently in malaria economic evaluations. The impact of variation in malaria risk on cost effectiveness can be, and often is, assessed by univariate or probabilistic sensitivity analysis. This risks conflating an important distinction between heterogeneity and uncertainty; it may be useful to the decision maker to understand both the differences between geographies and the degree of confidence in this information. Detailed stratification of cost-effectiveness results by disease risk would allow readers to match the characteristics of their context with study findings. However, geographic variation may include multiple variables preventing clear stratification.

Economic evaluation can do more to integrate information on geographic variability in situations where this is central to the policy response. There are several recent examples of studies that model geographic resource allocation for countries or regions in malaria and other disease areas [18-20]. Though aiming to reconcile costs and consequences, such studies are not always described as economic evaluations, often originating in the disciplines of mathematical modelling or spatial epidemiology. This paper describes an approach to geographic resource allocation grounded in economic evaluation methodology.

\section{Geographic Resource Allocation}

Healthcare economic evaluation models provide estimates of the cost and health impact for interventions of interest. Given relevant local data on demographics, disease risk and other factors for a set of geographically defined populations (hence 'geo-units'), such models can provide a menu or league table of interventions or intervention combinations in terms of their expected costs and effects. That is, a modelled estimate of the cost and effect of each intervention option in each geo-unit. The interpretation of such a dataset is not trivial, and the decision problem addressed in this paper is to select intervention options for each geo-unit such that health gains are maximised given the resources available.

A wide range of methods are available to model intervention costs and effects, including decision trees, compartmental or Markov models and individual-based models [21-23]. Some considerations in model design may include intervention interactions, the impact on disease transmission as well as health, the marginal effects of one intervention in the presence of another, variations over time and population behaviour, to name a few. Where multiple interventions are being considered, the model may need to be able to simulate their impact in isolation and in varying combinations. It is important to use an outcome measure that adequately captures the impact of all interventions of interest; utility metrics including the disability-adjusted life-year (DALY) or quality-adjusted life-year are appropriate, though some diseasespecific measures such as reduction in prevalence may be sufficient. Notwithstanding the above, all models are simplifications of reality, and the model should be no more complex than is necessary to reasonably estimate intervention costs and effects. The literature on modelling economic and health impacts is extensive. 
Box 1 Geographic resource allocation steps

The starting point of this method is a set of cost and effect estimates for all intervention options in all geographic units of interest. The aim is to select interventions and intervention bundles by geo-unit in such a way that impact is maximised for a given budget

1. Remove all interventions where there exists an alternative within the same geo-unit that is both more effective and less costly (absolute domination)

2. Calculate ICERs for all intervention combinations in all geounits using a common 'no additional intervention' comparator

3. Considering all remaining intervention options in all geo-units, select the option with the lowest ICER to allocate funding. Reduce the budget by the cost of this selection

4. If the selection displaces another intervention option in its geounit, then remove the displaced option from the league table and add its cost to the running budget

5. Recalculate the ICER for any remaining interventions in the selection geo-unit, using the newly selected intervention option as the comparator

6. Remove any intervention options where the ICER is negative (extended domination, the intervention is not absolutely dominated yet does not fall on the cost-effectiveness frontier and thus is not selected at any point)

7. Repeat steps 3-6 until the running budget is less than the cost of the next selection

ICER incremental cost-effectiveness ratio

\subsection{Allocation Method}

Geographic resource allocation is a variation of the knapsack problem ${ }^{1}$ and is analogous to priority setting between healthcare domains in standard healthcare priority setting using cost-effectiveness or cost-utility analysis [24]. The allocation problem can be stated as follows:

$\max \sum_{i \in I} \sum_{g \in G} e_{i g} d_{i g}$

s.t. $\sum_{i \in I} \sum_{g \in G} c_{i g} d_{i g} \leq b$

The sum of intervention effects $(e)$ is maximised subject to a budget constraint $(b)$ on the sum of intervention costs $(c)$, where the set of all interventions $(i)$ in all geo-units $(g)$ are included or excluded using a decision variable $(d)$. The decision variable determines inclusion or exclusion of intervention options and normally $d \in\{0,1\}$. Obtaining a solution to the optimisation problem is more tractable given an assumption of divisibility (see Sect. 2.3).

The allocation algorithm described here is an adaptation of priority setting using cost-effectiveness analysis rather

\footnotetext{
1 A standard combinatorial optimisation of placing objects of different weights into a knapsack.
}

than an iterative trial-and-improvement optimisation solver and is illustrated with a simplified example of a decision problem in three geographic units: A, B and C. Two interventions, ITNs and malaria CHWs, are available, as well as the combination of both interventions and the option to provide neither. Figure 1, panel 1, presents this example graphically, with information on the costs, effects and allocation results in Table 1. The corresponding costeffectiveness plane and the allocation result is presented in Fig. 1, panel 2, and the steps of the allocation algorithm are summarised in Box 1.

Starting with the costs and effect estimates for all intervention options in all geo-units, all dominated interventions (those that are more costly and less effective than an alternative intervention within the same geo-unit) are excluded. Incremental cost-effectiveness ratios (ICERs) are calculated for all interventions using the null or 'no additional intervention' scenario as the comparator. Priority setting is then broken down into a series of decisions. The first decision selects the intervention option with the lowest ICER from all non-excluded options in all geo-units. This selection is the choice that produces the maximum expected health gains for the investment (though it may be replaced by a subsequent selection in the same geo-unit). The cost of the intervention is subtracted from the budget, and the ICERs for the remaining interventions in the same geo-unit are recalculated using the selected intervention as the comparator (see Sect. 2.2). Any recalculated ICERs that are negative are subject to extended domination and excluded. These recalculated ICERs are those that form the cost-effectiveness frontier for the geo-unit. These steps are repeated, selecting the most cost-effective intervention option, recalculating the ICER and adjusting the budget. If the selection is in a geo-unit for which an intervention is already allocated, the new selection displaces the previous one and the cost of the displaced intervention is returned to the running budget. The process ends when the remaining budget is less than the cost of the next most cost-effective option.

Given the challenges specifying and interpreting costeffectiveness thresholds (CETs) for malaria control and elimination [25], the approach uses a budget as the resource constraint, which, because of the role of international aid, is often known and ring-fenced. Alternatively, if we instead wish to apply a CET as the resource constraint, each geounit can be treated as an isolated decision problem. As before, interventions subject to domination by others within the same geo-unit are excluded. Then, following standard economic evaluation methods, the cost-effectiveness frontier is identified and ICERs calculated sequentially along this frontier. The intervention option with the highest ICER below the CET is the expected optimal choice in each geounit. 


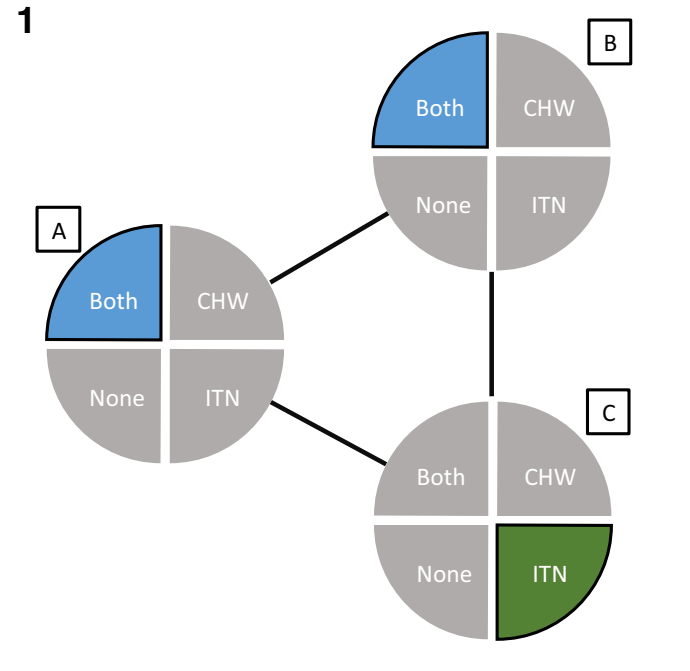

Fig. 1 Illustrative geographic allocation with cost-effectiveness plane (matching Table 1). Panel 1: $A, B$ and $C$ are illustrative geounits corresponding to the example described in Sect. 2.1 and Table 1.

Table 1 Example results table for multiple intervention resource allocation

\begin{tabular}{|c|c|c|c|c|c|c|c|}
\hline \multirow{2}{*}{$\begin{array}{l}\text { Geographic } \\
\text { unit }\end{array}$} & \multirow[t]{2}{*}{ Intervention } & \multirow{2}{*}{$\begin{array}{l}\text { Effect (DALYs } \\
\text { averted) }\end{array}$} & \multirow{2}{*}{$\begin{array}{l}\text { Cost } \\
(\$ U S)\end{array}$} & \multicolumn{2}{|c|}{ ICER (\$US per DALY averted) } & \multirow{2}{*}{$\begin{array}{l}\text { Allocation } \\
\text { result }^{b}\end{array}$} & \multirow{2}{*}{$\begin{array}{l}\text { Selection } \\
\text { rank }\end{array}$} \\
\hline & & & & $\begin{array}{l}\text { With null } \\
\text { comparator }\end{array}$ & $\begin{array}{l}\text { With frontier } \\
\text { comparator }^{\mathrm{a}}\end{array}$ & & \\
\hline \multirow[t]{3}{*}{ A } & ITN & 3.73 & 304 & 82 & 82 & Displaced & 1 \\
\hline & $\mathrm{CHW}$ & 3.4 & 380 & 112 & - & $\begin{array}{l}\text { Abs. } \\
\text { dominated }\end{array}$ & \\
\hline & Both & 5.01 & 498 & 99 & 152 & Funded & 3 \\
\hline \multirow[t]{3}{*}{ B } & ITN & 3.41 & 501 & 146 & 146 & Displaced & 2 \\
\hline & CHW & 3.62 & 733 & 202 & - & Ex. dominated & \\
\hline & Both & 4.29 & 804 & 187 & 344 & Funded & 5 \\
\hline \multirow[t]{3}{*}{$\mathrm{C}$} & ITN & 3.01 & 533 & 177 & 177 & Funded & 4 \\
\hline & CHW & 3.27 & 767 & 235 & 900 & Unfunded & \\
\hline & Both & 3.38 & 933 & 276 & 1509 & Unfunded & \\
\hline
\end{tabular}

Abs. absolutely, $C H W$ community health worker, $D A L Y$ disability-adjusted life-year, Ex. extended, ICER incremental cost-effectiveness ratio, ITN insecticide-treated bed net

a A by-product of the allocation process is the cost-effectiveness threshold or willingness to pay implied by the relevant budget. The threshold would fall between the least cost-effective intervention that is funded and the most cost-effective intervention that is not. In the example in Table 1, this is between \$US344 and \$US900 per DALY averted

b Given a budget constraint of \$US2000

\subsection{Incremental Cost-Effectiveness Ratio Calculation: Which Comparator?}

When evaluating intervention options across multiple geounits, identification of the appropriate comparator is essential to calculation of the correct ICER. Where a new intervention selection replaces a previous one, the difference in costs and effects between these alternatives should be used to decide whether the additional investment justifies the additional benefit. In this respect, there is a
The highlighted segments denote the intervention options selected to receive funding in the example. Abs. absolutely, $E x$. extended, $C H W$ community health worker, ITN insecticide-treated bed net 


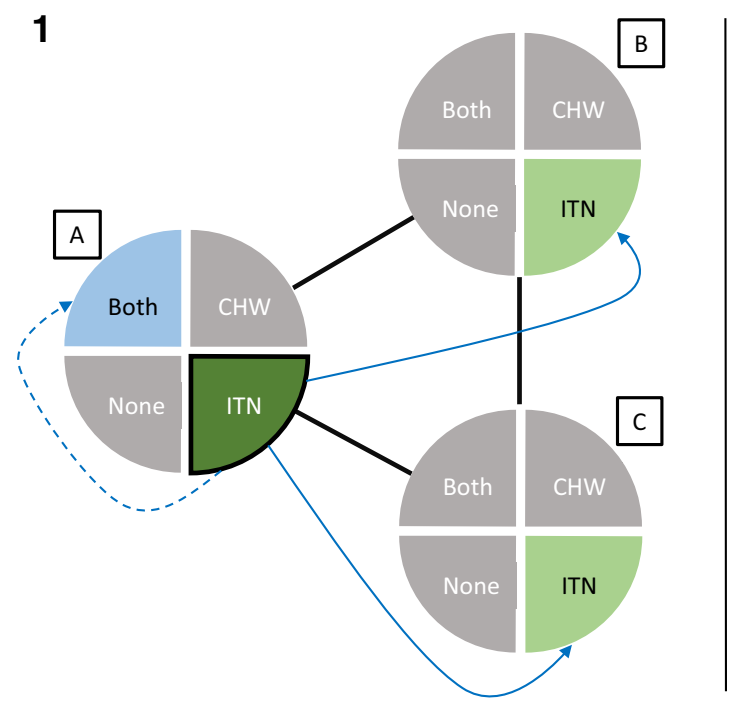

Fig. 2 Graphical representation of within and between geo-unit selection having initially selected ITN in geo-unit A. Panel 1: A, $B$ and $C$ are illustrative geo-units corresponding to the example within vs. between geo-unit selection described in Sect. 2.2. The darker highlighted ITN segment in geo-unit A denotes the intervention

difference between the costs and health gains between these interventions.

Calculation of the correct ICERs emerges in the process of the algorithm described in Sect. 2.1. At the point of selecting an intervention for inclusion, the ICERs for all remaining interventions within the same geo-unit are recalculated with the selected intervention as comparator, reflecting that, within a geo-unit, a new selection will replace the previous selection.

\subsection{Geo-Units}

To address a practical budget allocation problem, the geounits are likely to be pre-established administrative constituencies such as districts, townships or perhaps villages. The choice of administrative level should reflect how the interventions of interest may realistically be implemented. For example, for reasons of pragmatism and economies of scale, it may be more appropriate to allocate mass distribution of goods such as ITNs by district or township rather than by village. It is also essential that information such as population and disease risk must be available for the chosen administrative level. It may also be beneficial to include variable costs for different geo-units and for programme scale [26, 27].

Lastly, sequential selection based on ICERs does not necessarily provide a perfectly optimal solution for a discrete allocation problem in that there is a degree of error associated with the residual underspend [28]. In cost-effectiveness analysis, decision divisibility is assumed, allowing partial implementation of interventions. That is, selected in the first step; the lighter highlighted segments denote the three options available for selection in the second step. Panel 2 represents the corresponding cost-effectiveness plane. $\mathrm{CHW}$ community health worker, ITN insecticide-treated bed net

the decision variable $(d)$ in the knapsack objective function (Sect. 2) may take fractional values. While the assumption of divisibility in cost effectiveness has been debated [29-31], it is reasonable in the context of geographic targeting, where divisibility of intervention decisions by geographic areas is already assumed. Moreover, in the geographic allocation methods described here, the divisibility assumption is only necessary at the margin. That is, at the end of the allocation algorithm, it is assumed the most cost-effective unfunded intervention geo-unit option could be partially implemented using the remaining funds. It may not be necessary to formally include the partial unit allocation, as the interpretation of results would not reflect such precision (see Sect. 3.1), as in cost-effectiveness analysis, an underlying assumption of divisibility may be sufficient.

\subsection{Uncertainty}

Assessing and communicating the robustness of results given uncertainty in the model inputs is essential for rigorous economic evaluation. Probabilistic uncertainty can be incorporated into the allocation analysis by assigning sampling distributions to input variables and making repeated simulations of the allocation result (Monte Carlo simulation). A clear distinction can be made between this variation due to uncertainty and variation due to heterogeneity, and this is incorporated into the resource allocation analysis described above. The result of a probabilistic uncertainty analysis would be a probability score for each intervention in each geo-unit that it is the optimal choice 
given the defined parameter uncertainty. In such a probabilistic (or Bayesian) framework, additional decision criteria can be incorporated to reflect decision maker preferences such as aversion to overspend or undersupply $[32,33]$. Univariate or multivariate sensitivity analysis or scenario analysis can be used to assess parameter importance and scenarios of interest. Finally, structural uncertainty can be examined by repeating the allocation analysis with different underlying malaria models.

\subsection{Case Study: Universal Insecticide-Treated Bed Net Coverage vs. Targeted Resource Allocation in Myanmar}

A geographic resource allocation approach is employed by Drake et al. [19] to examine the cost effectiveness of ITN and malaria CHW in the Myanmar Artemisinin Resistance Containment (MARC) region [19]. Here, we use scenarios from the same context to illustrate the comparative advantage of targeted resource allocation over universal coverage (Fig. 3).

Based on the parameters detailed in Drake et al. [19], universal ITN coverage in the MARC region has an annual
A
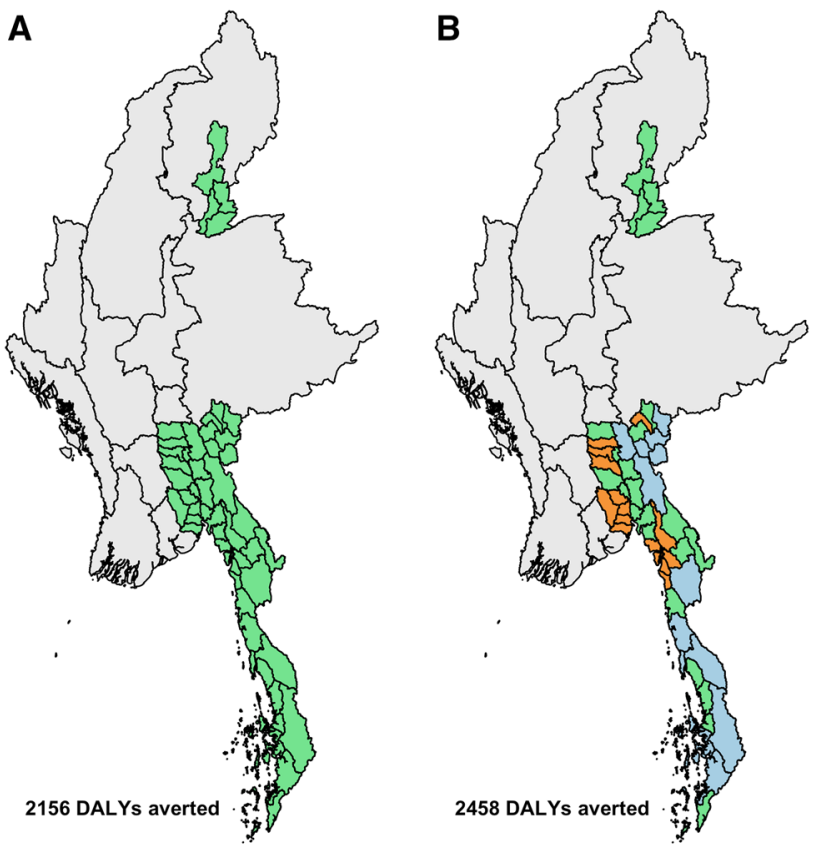

Intervention No Intervention
Fig. 3 Geographic allocation of budget for universal insecticide bed net coverage compared with targeting of both bed nets and community health workers. a Universal bed net coverage (within the MARC region) compared with $\mathbf{b}$ geographic targeting of both bed nets and community health workers. $C H W$ community health worker, $D A L Y$ disability-adjusted life-year, ITN insecticide-treated bed net, MARC Myanmar Artemisinin Resistance Containment equivalent cost of \$US6.08 million. The expected total impact of this compared with a hypothetical 'do nothing' scenario is 2156 DALYs averted. In comparison, the same budget allocated to ITNs, malaria CHWs or both by township on the basis of cost effectiveness yields a total impact of 2458 DALYs averted, a 14\% increase in impact compared with universal coverage in the first year without including reduction in transmission. It is worth noting that this is within a region already identified as high priority for malaria control efforts; application to the country as a whole or wider regions could yield greater efficiencies. Effectively targeted malaria interventions have the potential for greater impact than universal coverage of a single 'most cost-effective' intervention. This is already a common view in many policy circles. Economic analysis can do more to support geographic targeting in malaria control and elimination.

\section{Discussion}

This paper describes an approach for the geographic allocation of a given budget using locally specific estimates of the costs and effects of multiple interventions or intervention bundles. This framework is described in the context of malaria policy making but could be equally relevant in other disease contexts, such as schistosomiasis and soiltransmitted helminths [34] or HIV/AIDS [18].

\subsection{Interpretation}

A strength of this approach is that results are clear and directly applicable to the planning process, offering recommendations not only as to which interventions to invest in but where to target them to maximise health gains. Moreover, the recommendations are constrained by a relevant budget rather than a CET that, if too high, may recommend unaffordable policies or, if reflecting societal affordability constraints, may underestimate available resources because of international aid. In the context of malaria, budget-based geographic targeting can better reflect a real and timely decision problem than generalised threshold-based economic evaluations.

Nevertheless, a model is a simplification of a complex process and should be interpreted as such. All model inputs, the structure of the underlying model and the resource allocation framework are necessarily limited. While the model results may offer one recommendation, decision makers often have rich knowledge of the local context and should interpret model results as a potentially useful synthesis of relevant information that is nonetheless a simplification. 


\subsection{Alternative Methods}

In addition to the methods outlined in this paper, there are alternative approaches to geographic resource allocation. Walker et al. [20] applied a cost-minimisation framework and simulated a large number of different allocation options, choosing the least costly configuration that achieved a particular endpoint such as disease elimination. The approach differs from budget allocation in that it does not specify a resource constraint. A possible advantage of this or similar simulation approaches is that optimal solutions can be found for allocation problems without making the assumption of divisibility. A drawback of a simulation approach is the substantial computation time required, notwithstanding techniques to improve efficiency, such as simulated annealing. Computation time for the approach described in this paper is negligible and therefore dependent only on the underlying cost-effectiveness model. For simple models, probabilistic sensitivity analysis and interactive user interfaces can be possible, both of which are potentially valuable extensions.

\subsection{Limitations}

There are several limitations to the resource allocation framework described in this paper.

First, all limitations of the underlying model used to estimate intervention costs and effects apply equally to the allocation result. Care needs to be taken when comparing allocation results from different models because characteristics or artefacts of the underlying model could drive important differences in results. Second, the method objective is a simple maximisation of health utility. It may be beneficial in some contexts to extend the approach to include non-health benefits such as financial protection [35] or to incorporate equity preferences [36]. Third, the friction cost of administering targeted, as opposed to universal, healthcare programmes is not included, and there may be differences in economies of scale. Incorporation of scale into a variable cost function would be a valuable addition. Lastly, the allocation results can only be as good at the input data. Reliable locally specific information on disease risk and other variables, such as treatment-seeking behavior or intervention cost, are essential and not always readily available. In particular, the potential bias of under-reporting disease burden in areas with poor health surveillance is problematic for any geographic targeting based on such data, whether in a formal allocation framework or otherwise.

\section{Conclusion}

Geographic targeting is an increasingly common feature of malaria control and elimination strategies but does not feature prominently in malaria economic evaluations. This paper describes a framework for geographic budget allocation grounded in economic evaluation methods. Efficient targeting of core malaria interventions has the potential to increase the impact of the resources available, accelerating progress towards disease elimination. These methods will also be applicable to priority setting in other disease areas.

Acknowledgements The authors thank Boby Mihaylova and Zaid Chalabi for their valuable comments on early drafts of the manuscript and Patrick Walker, Peter Winskill and colleagues for stimulating discussion of geographic resource allocation methods.

Author contributions TD, YL and LW developed the geographic allocation method. TD, YL, SSK, MPK and LW developed the case study. AD, FS and ND contributed to manuscript development and communication of the method. All authors read and contributed to the final manuscript.

\section{Compliance with Ethical Standards}

Ethical approval for modelling analysis using Myanmar incidence data was obtained from the Department of Medical Research, Yangon, Myanmar and the Oxford Tropical Research Ethics Committee, University of Oxford, UK.

Conflict of interest The authors TD, YL, SSK, AD, MPK, ND, FS and LW have no conflicts of interest.

Funding TD, YL, SSK and LW are supported by a grant from the Bill and Melinda Gates Foundation. The Mahidol Oxford Tropical Medicine Research Unit is supported by the Wellcome Trust major overseas programme in South East Asia (Grant Number 106698/Z/14/ Z).

Open Access This article is distributed under the terms of the Creative Commons Attribution-NonCommercial 4.0 International License (http://creativecommons.org/licenses/by-nc/4.0/), which permits any noncommercial use, distribution, and reproduction in any medium, provided you give appropriate credit to the original author(s) and the source, provide a link to the Creative Commons license, and indicate if changes were made.

\section{References}

1. Alonso PL, Tanner M. Public health challenges and prospects for malaria control and elimination. Nat Med. 2013;19:150-5.

2. White MT, Conteh L, Cibulskis R, Ghani AC. Costs and costeffectiveness of malaria control interventions: a systematic review. Malar J. 2011;10:337.

3. Goodman C, Coleman P, Mills A. Cost-effectiveness of malaria control in sub-Saharan Africa. Lancet. 1999;354:378-85.

4. Goodman CA, Mills AJ. The evidence base on the cost-effectiveness of malaria control measures in Africa. Health Policy Plan. 1999;14:301-12.

5. Lubell Y, Riewpaiboon A, Dondorp AM, et al. Cost-effectiveness of parenteral artesunate for treating children with severe malaria in sub-Saharan Africa. Bull World Health Organ. 2011;89:504-12.

6. Shillcutt S, Morel C, Goodman C, Coleman P, Bell D, Whitty CJ, Mills A. Cost-effectiveness of malaria diagnostic methods in subSaharan Africa in an era of combination therapy. Bull World Health Organ. 2008;86:101-10. 
7. Mangham-Jefferies L, Wiseman V, Achonduh OA, Drake TL, Cundill B, Onwujekwe O, Mbacham W. Economic evaluation of a cluster randomized trial of interventions to improve health workers' practice in diagnosing and treating uncomplicated malaria in Cameroon. Value Health. 2014;17:783-91.

8. Lubell Y, Reyburn H, Mbakilwa H, Mwangi R, Chonya K, Whitty CJM, Mills A. The cost-effectiveness of parasitologic diagnosis for malaria-suspected patients in an era of combination therapy. Am J Trop Med Hyg. 2007;77:128-32.

9. Gething PW, Patil AP, Smith DL, Guerra CA, Elyazar IR, Johnston GL, Tatem AJ, Hay SI. A new world malaria map: Plasmodium falciparum endemicity in 2010. Malar J. 2011;10:378.

10. Gething PW, Elyazar IRF, Moyes CL, et al. A long neglected world malaria map: Plasmodium vivax endemicity in 2010. PLoS Negl Trop Dis. 2012;6:e1814.

11. Dalrymple U, Mappin B, Gething PW. Malaria mapping: understanding the global endemicity of falciparum and vivax malaria. BMC Med. 2015;13:140.

12. Bejon P, Williams TN, Nyundo C, et al. A micro-epidemiological analysis of febrile malaria in Coastal Kenya showing hotspots within hotspots. eLife. 2014;3:e02130.

13. Wesolowski A, Eagle N, Tatem AJ, Smith DL, Noor AM, Snow RW, Buckee CO. Quantifying the impact of human mobility on malaria. Science. 2012;338:267-70.

14. Pindolia DK, Garcia AJ, Huang Z, Smith DL, Alegana VA, Noor AM, Snow RW, Tatem AJ. The demographics of human and malaria movement and migration patterns in East Africa. Malar J. 2013;12:397.

15. Bousema T, Stevenson J, Baidjoe A, et al. The impact of hotspottargeted interventions on malaria transmission: study protocol for a cluster-randomized controlled trial. Trials. 2013;14:36.

16. Wangdi K, Banwell C, Gatton ML, Kelly GC, Namgay R, Clements ACA. Development and evaluation of a spatial decision support system for malaria elimination in Bhutan. Malar J. 2016;15:180.

17. Marston L, Kelly GC, Hale E, Clements ACA, Hodge A, Jimenez-Soto E. Cost analysis of the development and implementation of a spatial decision support system for malaria elimination in Solomon Islands. Malar J. 2014;13:325.

18. Anderson S-J, Cherutich P, Kilonzo N, et al. Maximising the effect of combination HIV prevention through prioritisation of the people and places in greatest need: a modelling study. Lancet. 2014;384:249-56.

19. Drake TL, Kyaw SS, Kyaw MP, Smithuis FM, Day NP, White LJ, Lubell Y. Cost effectiveness and resource allocation of Plasmodium falciparum malaria control in Myanmar: a modelling analysis of bed nets and community health workers. Malar J. 2015; 14:376.

20. Walker PGT, Griffin JT, Ferguson NM, Ghani AC. Estimating the most efficient allocation of interventions to achieve reductions in Plasmodium falciparum malaria burden and transmission in Africa: a modelling study. Lancet Glob Health. 2016;4:e474-84.

21. Drummond MF, Sculpher MJ, Torrance GW. Methods for the economic evaluation of health care programs. Oxford: Oxford University Press; 2005.

22. Briggs A. Probabilistic analysis of cost-effectiveness models: statistical representation of parameter uncertainty. Value Health. $2005 ; 8: 1-2$.

23. Gray AM, Clarke PM, Wolstenholme JL, Wordsworth S. Applied methods of cost-effectiveness analysis in healthcare. Oxford: Oxford University Press; 2011.

24. Weinstein M, Zeckhauser R. Critical ratios and efficient allocation. J Public Econ. 1973;2:147-57.

25. Drake T, Lubell Y. Malaria and economic evaluation methods: Challenges and opportunities. Appl Health Econ Health Policy 2017. doi:10.1007/s40258-016-0304-8

26. Meyer-Rath G, Over M. HIV treatment as prevention: modelling the cost of antiretroviral treatment-state of the art and future directions. PLOS Med. 2012;9:e1001247.

27. Lépine A, Chandrashekar S, Shetty G, Vickerman P, Bradley J, Alary M, Moses S, CHARME India Group, Vassall A. What determines HIV prevention costs at scale? Evidence from the Avahan programme in India. Health Econ. 2016;25(Suppl 1):67-82.

28. Bennett JP, Saaty TL. Knapsack allocation of multiple resources in benefit-cost analysis by way of the analytic hierarchy process. Math Comput Model. 1993;17:55-72.

29. Birch S, Gafni A. Changing the problem to fit the solution: Johannesson and Weinstein's (mis) application of economics to real world problems. J Health Econ. 1993;12:469-76.

30. Johannesson M, Weinstein MC. On the decision rules of costeffectiveness analysis. J Health Econ. 1993;12:459-67.

31. Gafni A, Birch S. Incremental cost-effectiveness ratios (ICERs): the silence of the lambda. Soc Sci Med. 2006;62:2091-100.

32. Chalabi Z, Epstein D, McKenna C, Claxton K. Uncertainty and value of information when allocating resources within and between healthcare programmes. Eur J Oper Res. 2008;191: 530-9.

33. McKenna C, Chalabi Z, Epstein D, Claxton K. Budgetary policies and available actions: a generalisation of decision rules for allocation and research decisions. J Health Econ. 2010;29:170-81.

34. Lo NC, Lai Y-S, Karagiannis-Voules D-A, Bogoch II, Coulibaly JT, Bendavid E, Utzinger J, Vounatsou P, Andrews JR. Assessment of global guidelines for preventive chemotherapy against schistosomiasis and soil-transmitted helminthiasis: a cost-effectiveness modelling study. Lancet Infect Dis. 2016;16:1065-75.

35. Verguet S, Laxminarayan R, Jamison DT. Universal public finance of tuberculosis treatment in India: an extended cost-effectiveness analysis. Health Econ. 2015;24:318-32.

36. Cookson R, Drummond M, Weatherly H. Explicit incorporation of equity considerations into economic evaluation of public health interventions. Health Econ Policy Law. 2009;4:231-45. 